\title{
Focal Osteolysis at the Junctions of a Modular Stainless-STeel FEMORAL INTRAMEDULlary NAIL
}

\author{
By Darron M. Jones, MD, J. LaWrence Marsh, MD, James V. Nepola, MD, Joshua J. Jacobs, MD, \\ ANASTASia K. Skipor, MS, Robert M. Urban, Jeremy L. Gilbert, PhD, AND JOSEPH A. BuckWALTER, MD \\ Investigation performed at the University of Iowa Hospitals and Clinics, Iowa City, Iowa, \\ Rush Arthritis and Orthopaedics Institute, Rush-Presbyterian-St. Luke's Medical Center, Chicago, Illinois, \\ and Department of Bioengineering and Neuroscience, Syracuse University, Syracuse, New York
}

\begin{abstract}
Background: During routine follow-up of patients treated with a three-piece stainless-steel modular femoral nail, osteolysis and periosteal reaction around the modular junctions of some of the nails were noted on radiographs. The purpose of this study was to evaluate the prevalence, etiology, and clinical relevance of these radiographic findings.

Methods: Forty-four femoral fractures or nonunions in forty-two patients were treated with a modular stainlesssteel femoral intramedullary nail. Seventeen nails were excluded, leaving twenty-seven intramedullary nails in twenty-seven patients for this study. All patients had had a femoral diaphyseal fracture; nineteen had had an acute fracture and eight, a nonunion. These twenty-seven patients returned for radiographs, a physical examination, assessment of functional outcomes, assessment of thigh pain with a visual analog scale, determination of serum chromium levels, and nail removal if desired. A control group of sixteen patients treated with a one-piece stainless-steel femoral intramedullary nail was evaluated with use of the same outcome measures and was compared with the group treated with the modular femoral nail with regard to prevalence of thigh pain and serum chromium levels. Twelve modular femoral nails were removed according to the study protocol. The modular nail junctions were analyzed for corrosion products, and histopathologic analysis of tissue specimens from the femoral canal was performed.

Results: The twenty-seven patients were seen at a mean of twenty-one months after fracture fixation; twenty-six of the twenty-seven fractures healed. Twenty-three femora had at least one of three types of abnormalities —osteolysis, periosteal reaction, or cortical thickening - localized to one or both modular junctions. Eighteen patients had severe reactions, defined as osteolysis of $\geq 2 \mathrm{~mm}$, cortical thickening of $\geq 5 \mathrm{~mm}$, and/or a periosteal reaction (group 1). Nine patients had mild or no reactions (group 2). Serum chromium levels in group 1 (mean, $1.27 \mathrm{ng} /$ $\mathrm{mL}$; range, 0.34 to $3.12 \mathrm{ng} / \mathrm{mL}$ ) were twice as high as those in group 2 (mean, $0.53 \mathrm{ng} / \mathrm{mL}$; range, 0.12 to 1.26 $\mathrm{ng} / \mathrm{mL}$ ). However, this difference did not reach significance with the numbers available. The differences in serum chromium levels between group 1 and the control group with a one-piece nail (mean, $0.26 \mathrm{ng} / \mathrm{mL}$; range, 0.015 to $1.25 \mathrm{ng} / \mathrm{mL})(p<0.01$ ) and a control group without an implant (mean, $0.05 \mathrm{ng} / \mathrm{mL}$; range, 0.015 to $0.25 \mathrm{ng}$ / $\mathrm{mL})(\mathrm{p}<0.01)$ were significant. The level of thigh pain recorded on the visual analog scale was also significantly different between group 1 and the control group with a one-piece implant $(p=0.03)$. Retrieved modular nails had signs of fretting corrosion as well as stainless-steel corrosion products adherent to the junction where the osteolysis occurred. Histologic and spectrographic analysis revealed two types of corrosion products that were consistent with stainless-steel within the peri-implant tissue and were associated with a foreign-body granulomatous response.

Conclusions: The presence of corrosion products at the taper junctions suggests that particulate debris was a major factor in the etiology of the radiographic findings of osteolysis, periosteal reaction, and cortical thickening. Serum chromium levels were substantially elevated in the patients with a modular femoral nail, and such levels may serve as a marker of fretting corrosion of these devices.
\end{abstract}

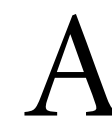

three-piece modular 316L stainless-steel femoral nail that is assembled intraoperatively was designed to decrease hospital inventory and cost. Different proximal and distal modular components permit standard antegrade nailing, retrograde nailing, or reconstruction nailing. Assem- bling the nail creates two tapered junctions where the two modular end components attach to the common central shaft. A large end attached to the insertion device and a smaller tapered end allow static interlocking and intraoperative adjustment of length. These ends are connected to the common 


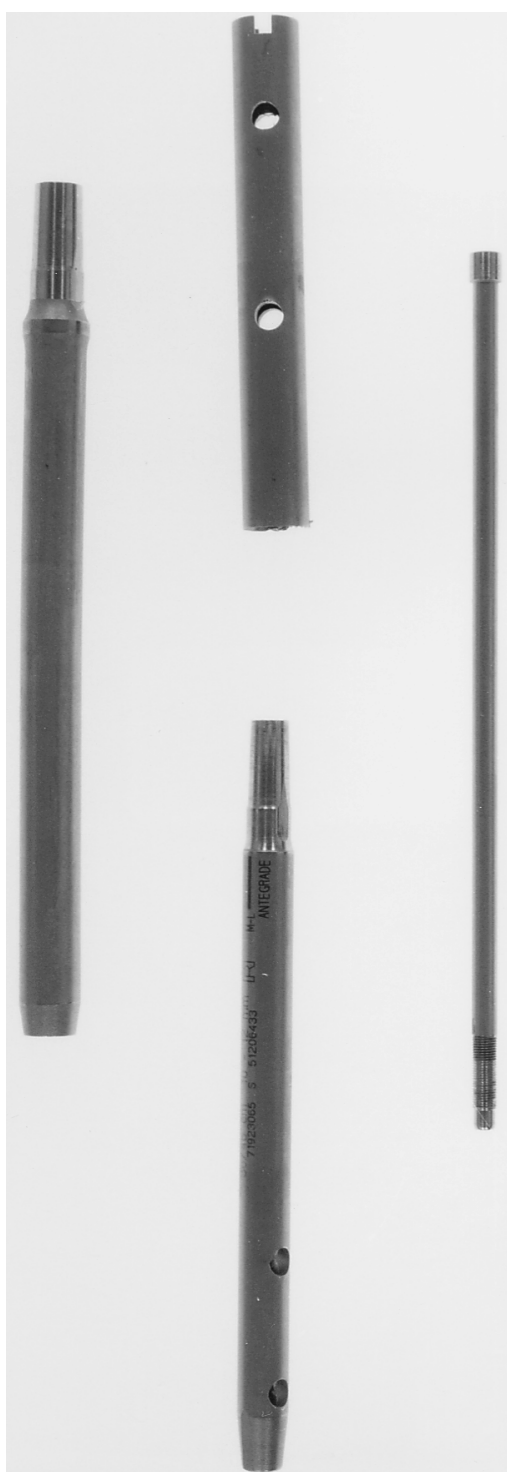

Fig. 1-A

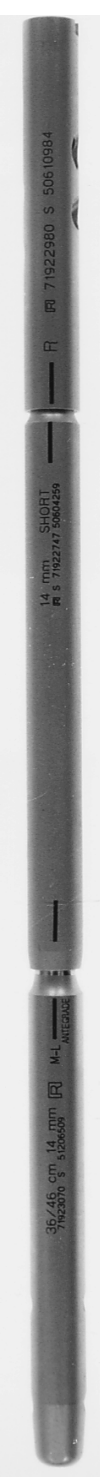

Fig. 1-B

Figs. 1-A and 1-B Photographs of the three-piece femoral modular nail. Fig. 1-A Prior to assembly. At the top is the large component that attaches to the insertion device. Below is the smaller, tapered component. The common central shaft is to the left, and the central draw bolt is to the right. Fig. 1-B After assembly. The large junction is at the top, and the small junction is at the bottom.

central shaft, by an interference fit of a truncated cone and bore, with a torque device that provides $6282 \mathrm{~N}$ of compressive force. The ends are then secured with a central draw bolt. In this paper, we refer to the modular junctions as large and small on the basis of the size of the component that attaches to the common central shaft (Figs. 1-A and 1-B). Therefore, with an antegrade nail, the large junction is proximal, in the subtrochanteric region, and the small junction is distal. With retrograde insertion, the large junction is distal and the small junction is proximal, in the subtrochanteric area. On manufacturer testing of the modular nail taper in torsional fatigue with a torsional load cycled between 25 and 250 in-lb (2.8 and

28.3 Nm), 2,000,000 cycles were completed, indicating high resistance to torsional fatigue.

Two patients treated with this nail presented, after union of the fracture, with thigh pain and osteolysis of the femur adjacent to one of the junctions ${ }^{1}$. An informal review of radiographs revealed that other patients had osteolysis and periosteal reaction in the femur adjacent to a modular nail junction. We hypothesized that patients with a modular femoral nail would have a greater prevalence of osteolytic reactions, higher serum chromium levels, and more thigh pain than a similar group of patients treated with a standard one-piece intramedullary femoral nail and that these findings are a result of particulate corrosion products formed at the junctions of the modular femoral nail. To test this hypothesis and to determine the prevalence, severity, and clinical relevance of osteolysis related to the presence of modular junctions, we performed a clinical and radiographic evaluation of all of our patients treated with this device and a group of control patients treated with a one-piece stainless-steel femoral nail. We attempted to define an association of the clinical and radiographic features with serum chromium levels, and we studied the retrieved nails and peri-implant tissue for the presence of corrosion and corrosion products.

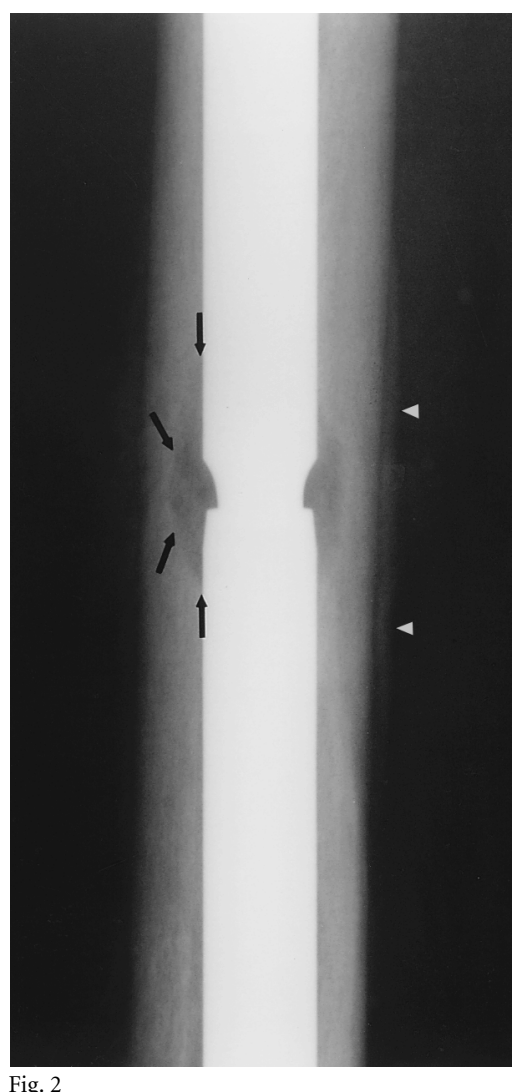

Case 21. Radiograph made four months following treatment of an acute fracture with a retrograde intramedullary nail, showing osteolysis and a periosteal reaction. The arrows depict the method for measuring osteolysis from the endosteal surface to the edge of the hemielliptical area. The arrowheads show periosteal reaction. 
The Journal of Bone \& Joint Surgery - JbJs.org $\quad$ Volume 83-A · Number 4 - April 2001

TABLE I Data on Patients Treated with a Modular Femoral Nail

\begin{tabular}{|c|c|c|c|c|c|c|c|c|c|c|}
\hline Case & Gender & $\begin{array}{l}\text { Age } \\
(y r)\end{array}$ & $\begin{array}{l}\text { Duration of } \\
\text { Follow-up } \\
(\mathrm{mo})\end{array}$ & $\begin{array}{l}\text { Fracture } \\
\text { Type* }\end{array}$ & $\begin{array}{l}\text { Open or } \\
\text { Closed } \dagger\end{array}$ & Method & $\begin{array}{l}\text { Nail } \\
\text { Size } \neq\end{array}$ & Interlocking & $\begin{array}{c}\text { Pain } \\
\text { Score§\# }\end{array}$ & $\begin{array}{c}\text { Serum } \\
\text { Chromium } \\
\text { Level\# } \\
(n g / m L)\end{array}$ \\
\hline 1 & M & 23 & 27 & 32 B3.2 & $\begin{array}{l}\text { Open, } \\
\text { grade II }\end{array}$ & Antegrade & $12 \times 38$ & Static & 0 & 0.2769 \\
\hline 2 & $F$ & 27 & 19 & 32 В3.2 & $\begin{array}{l}\text { Open, } \\
\text { grade II }\end{array}$ & Retrograde & $12 \times 42$ & Static & 2 & 0.6718 \\
\hline 3 & $M$ & 40 & 16 & Nonunion & Closed & Retrograde & $12 \times 34$ & Static & 0 & 0.4694 \\
\hline 4 & M & 26 & 24 & Nonunion & Closed & Retrograde & $12 \times 45$ & Static & 2 & 0.6388 \\
\hline 5 & $F$ & 35 & 22 & 32 A3.2 & Closed & Retrograde & $10 \times 32$ & Static & 5 & 0.5436 \\
\hline 6 & $F$ & 37 & 25 & 32 В3.3 & Closed & Retrograde & $10 \times 44$ & Static & 1 & 0.3353 \\
\hline 7 & $F$ & 37 & 19 & Nonunion & Closed & Antegrade & $12 \times 42$ & Dynamic & NA & 3.1150 \\
\hline 8 & M & 21 & 18 & 32 B2.3 & Closed & Retrograde & $12 \times 38$ & Static & 0 & 1.5150 \\
\hline 9 & M & 19 & 29 & 32 B2.2 & Closed & Antegrade & $12 \times 36$ & Dynamic & 0 & 1.2410 \\
\hline 10 & M & 42 & 27 & 32 B3.2 & Closed & Antegrade & $12 \times 45$ & Static & 0 & 0.2550 \\
\hline 11 & $F$ & 54 & 22 & 32 A3.2 & Closed & Retrograde & $12 \times 38$ & Static & 6 & 1.8078 \\
\hline 12 & $\mathrm{~F}$ & 39 & 18 & 32 A2.2 & Closed & Retrograde & $10 \times 32$ & Static & 5 & 1.6520 \\
\hline 13 & $\mathrm{~F}$ & 20 & 20 & Nonunion & Closed & Antegrade & $10 \times 36$ & Static & 5 & 0.9381 \\
\hline 14 & M & 21 & 28 & Nonunion & Closed & Antegrade & $14 \times 42$ & Static & 4 & 0.6293 \\
\hline 15 & M & 28 & 22 & Nonunion & Closed & Antegrade & $14 \times 44$ & Dynamic & 5 & 1.0810 \\
\hline 16 & $\mathrm{~F}$ & 25 & 27 & $32 \mathrm{C} 2.2$ & Closed & Antegrade & $12 \times 40$ & Static & 4 & 1.0336 \\
\hline 17 & M & 21 & 19 & 32 B3.2 & Closed & Antegrade & $12 \times 40$ & Static & 0 & NA \\
\hline 18 & M & 35 & 20 & 32 C1.2 & Closed & Retrograde & $14 \times 42$ & Static & 0 & 1.6300 \\
\hline 19 & $\mathrm{~F}$ & 31 & 12 & Nonunion & Closed & Retrograde & $12 \times 38$ & Static & 4 & 2.5360 \\
\hline 20 & M & 20 & 15 & 32 C2.2 & Closed & Antegrade & $14 \times 36$ & Static & 0 & 1.2552 \\
\hline 21 & M & 51 & 15 & 32 A1.3 & Closed & Retrograde & $14 \times 34$ & Static & 0 & 0.5738 \\
\hline 22 & M & 34 & 15 & 32 B2.3 & $\begin{array}{l}\text { Open, } \\
\text { grade III }\end{array}$ & Antegrade & $14 \times 42$ & Static & 4 & 1.4196 \\
\hline 23 & M & 30 & 16 & 32 A3.2 & Closed & Antegrade & $12 \times 38$ & Static & NA & NA \\
\hline 24 & M & 27 & 24 & 32 A3.2 & Closed & Retrograde & $12 \times 46$ & Static & 5 & 0.1571 \\
\hline 25 & $F$ & 29 & 22 & 32 C3.2 & $\begin{array}{l}\text { Open, } \\
\text { grade II }\end{array}$ & Retrograde & $14 \times 44$ & Static & 0 & 0.1247 \\
\hline 26 & $F$ & 42 & 27 & Nonunion & Closed & Antegrade & $12 \times 42$ & Dynamic & 3 & 1.0950 \\
\hline 27 & $F$ & 39 & 19 & 32 B3.2 & Closed & Antegrade & $12 \times 38$ & Static & 0 & 0.9112 \\
\hline
\end{tabular}

*According to the OTA (Orthopaedic Trauma Association) classification ${ }^{3}$. †The grade of the open fractures was determined with use of the

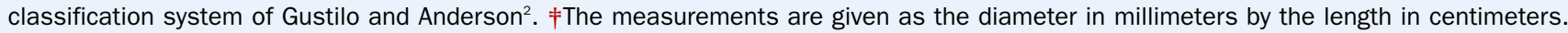
$\S$ The pain score was determined on a visual analog scale of 0 to 10 . \#NA = not available.

\section{Materials and Methods}

B etween September 1995 and December 1996, forty-four B femora in forty-two patients were treated at the University of Iowa Hospitals and Clinics with a Food and Drug Administration-approved modular 316L stainless-steel femoral nail (TriMax; Smith and Nephew, Memphis, Tennessee) as part of a clinical evaluation of this device, which had been released to a limited number of centers. The diagnosis was an acute fracture in twenty-eight patients, a nonunion in eight, and an impending pathologic fracture in eight.
We excluded the eight nails inserted to prevent impending pathologic fracture because those patients had osteolysis secondary to tumor. Another nail was excluded because it was associated with infection. It was removed two weeks after insertion from a patient who had been treated for a gunshot wound in the femur. Another modular femoral nail, which had been placed through a retrograde approach, was excluded because it broke at the proximal junction three months postoperatively. It was exchanged for a second, larger modular femoral nail, and this nail was included in the study. A third 


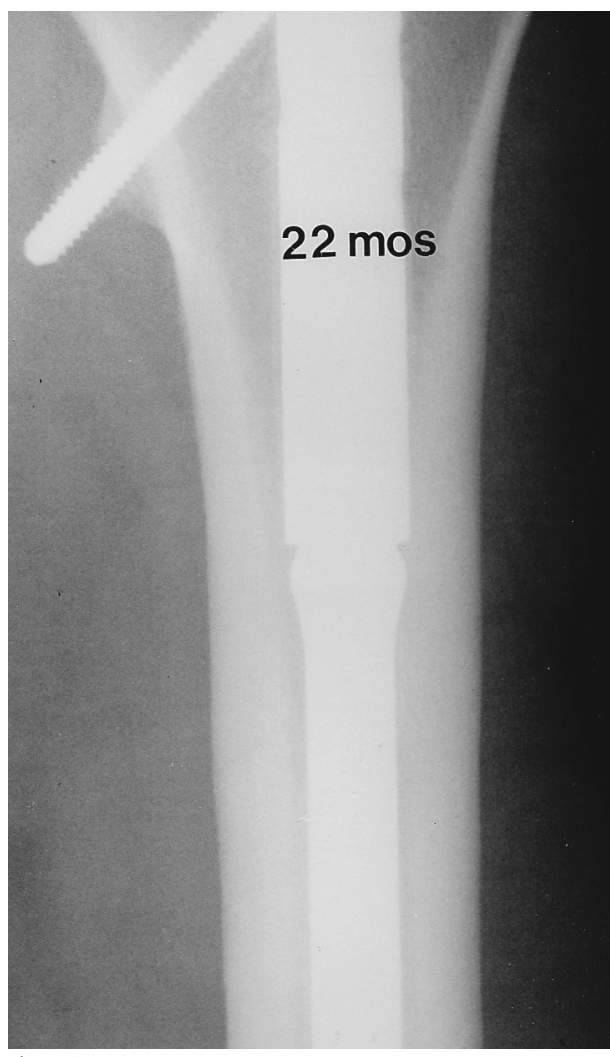

Fig. 3-A

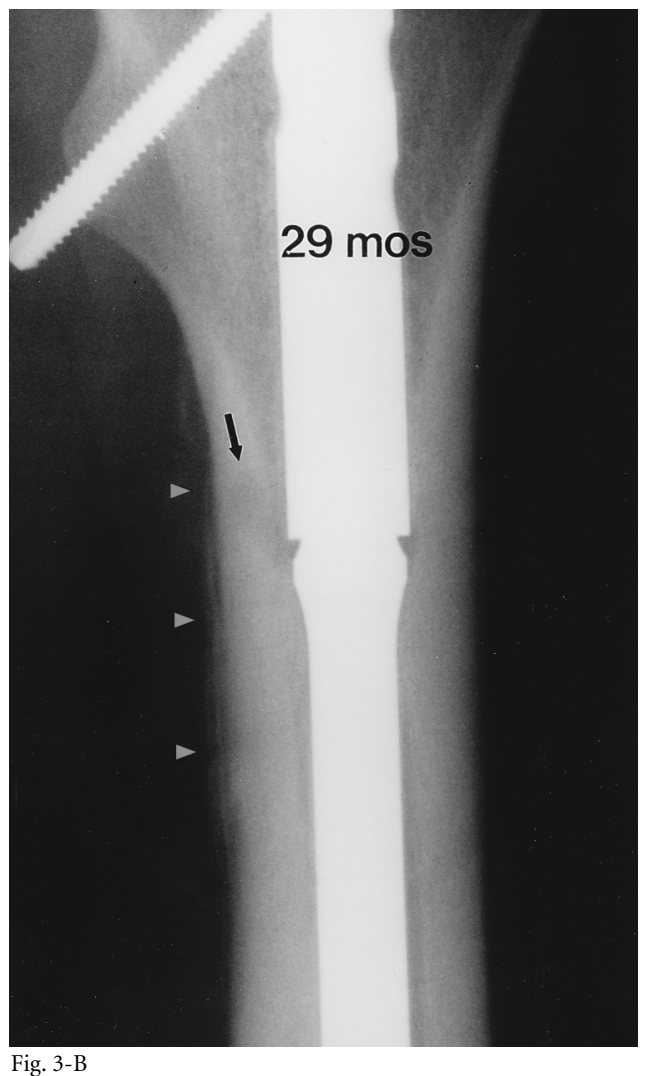

Fig. 3-B

Figs. 3-A and 3-B Case 9. Example of osteolysis and periosteal reaction adjacent to the large, proximal junction in a nail inserted through an antegrade approach. Fig. 3-A Radiograph made twenty-two months postoperatively, showing no findings at the junction. Fig. 3-B Radiograph made at twenty-nine months, showing a large area of osteolysis (arrow) and a periosteal reaction (arrowheads).

patient, who underwent exchange nailing with a standard Russell-Taylor nail (Smith and Nephew) at eleven months because of a nonunion, was also excluded. Finally, a patient from whom a modular femoral nail was routinely removed at another hospital was excluded.

This left thirty-two intramedullary modular femoral nails in thirty-two patients. Following approval from our institutional review board, each of these patients was sent a letter inviting them to return for radiographs, a physical examination, assessment of functional outcomes, evaluation of thigh pain with a visual analog scale, determination of serum chromium levels, and nail removal if desired. All patients signed an informed-consent form to participate in the study, and all were counseled regarding the osteolytic reactions and their possible clinical importance. The costs of the clinic visit and nail removal were covered by the orthopaedic department, the hospital, and the manufacturer. Five patients refused the invitation to return, so twenty-seven patients (nineteen with an acute fracture and eight with a nonunion) were evaluated at a mean of twenty-one months (range, twelve to twenty-nine months) following the index operation. Fourteen procedures were performed through an antegrade approach and thirteen, through an intra-articular retrograde approach. There were fifteen men and twelve women with a mean age of thirty-two years (range, nineteen to fifty-four years). Ten right and seventeen left femora were involved. Twenty-three fractures were closed, and four were open. With use of the Gustilo and Anderson system for open fractures ${ }^{2}$, three fractures were classified as grade II and one, as grade III. The nail was statically locked in twenty-three of the twentyseven fractures and in all but one of the acute fractures. Twenty-six of the twenty-seven fractures and nonunions healed. One patient (Case 23) had a nonunion after the femoral nailing; this healed following exchange nailing (Table I).

Serial radiographs were evaluated by two observers (D.M.J. and J.L.M.), who began with the radiographs of the injuries. With use of the Orthopaedic Trauma Association classification for femoral fractures ${ }^{3}$, six of the nineteen acute fractures were classified as OTA 32A; nine, as OTA 32B; and four, as OTA 32C. The eight nonunions, which included six hypertrophic and two atrophic nonunions, were treated at a mean of sixteen months (range, three to thirty months) following the injury. Seven of the eight patients had been treated previously with an intramedullary nail, and one had been treated with a plate and screws.

The location of the fracture in relation to the modular junctions, the tightest isthmal cortical fit (measured from the outer border of the nail to the inner cortex), and the distance between the junctions of the nail and the inner border of the cortex (measured to the nearest millimeter) were determined 
The Journal of Bone \& Joint Surgery - JbJs.org $\quad$ Volume 83-A · Number 4 - April 2001

TABLE II Radiographic Findings in Patients Treated with a Modular Femoral Nail

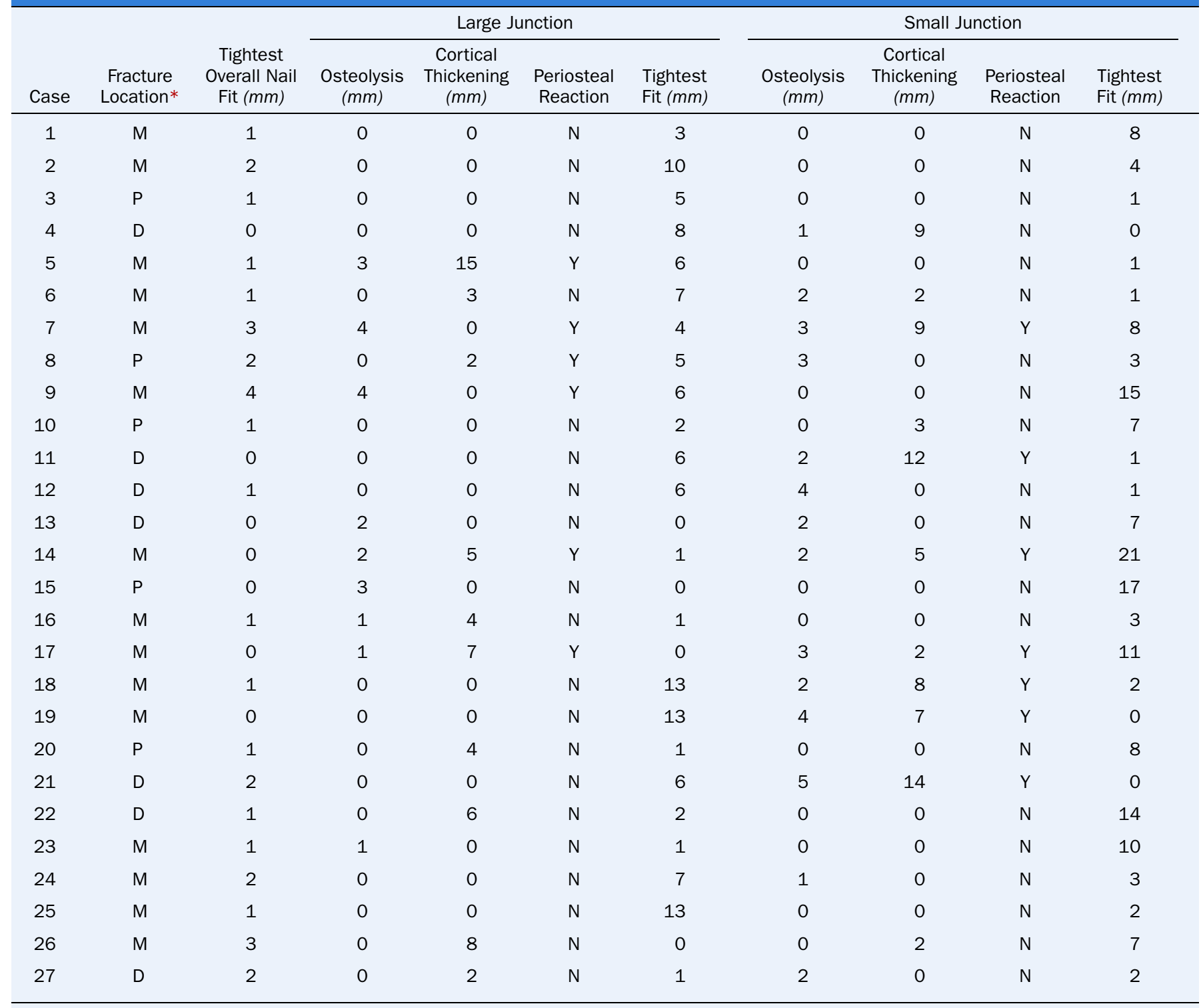

$* \mathrm{M}=$ middle of nail, $\mathrm{P}=$ proximal junction, and $\mathrm{D}=$ distal junction.

on the initial postoperative radiographs for all patients. The fracture was located at the level of one of the junctions in twelve patients, and these junctions were excluded from further analysis.

Three types of reactions-osteolysis, periosteal reaction, and cortical thickening-were observed radiographically at the levels of the junctions. Osteolysis was defined as a focal, hemielliptical area of rarefaction at the endosteal cortex. The extent of osteolysis was quantified by measuring the distance, in millimeters, from the endosteal cortex to the most medial or lateral extent of the radiolucency along a line perpendicular to the long axis of the femur (Fig. 2). Cortical thickening was determined by subtracting the greatest width of the cortex at the level of the junction on the immediate postoperative radiograph from the same measurement made on the follow-up radiograph. Periosteal reaction was recorded as either present or absent. The time of appearance of the radiographic findings and their location (at the large or small junction) were recorded.

At the time of the follow-up visit, each patient had an examination of the lower extremities. Twenty-five patients completed the Musculoskeletal Function Assessment instrument ${ }^{4,5}$ and marked a visual analog scale for thigh pain, on which 0 indicated no pain and 10 indicated severe, constant pain. Two patients had the nail removed before the time of the evaluation with the assessment form and the pain scale. Blood samples from twenty-five patients were assayed for serum chromium concentration with use of graphite furnace Zeeman atomic absorption spectrophotometry according to previously described protocols ${ }^{6}$. The detection limit for chromium in serum 


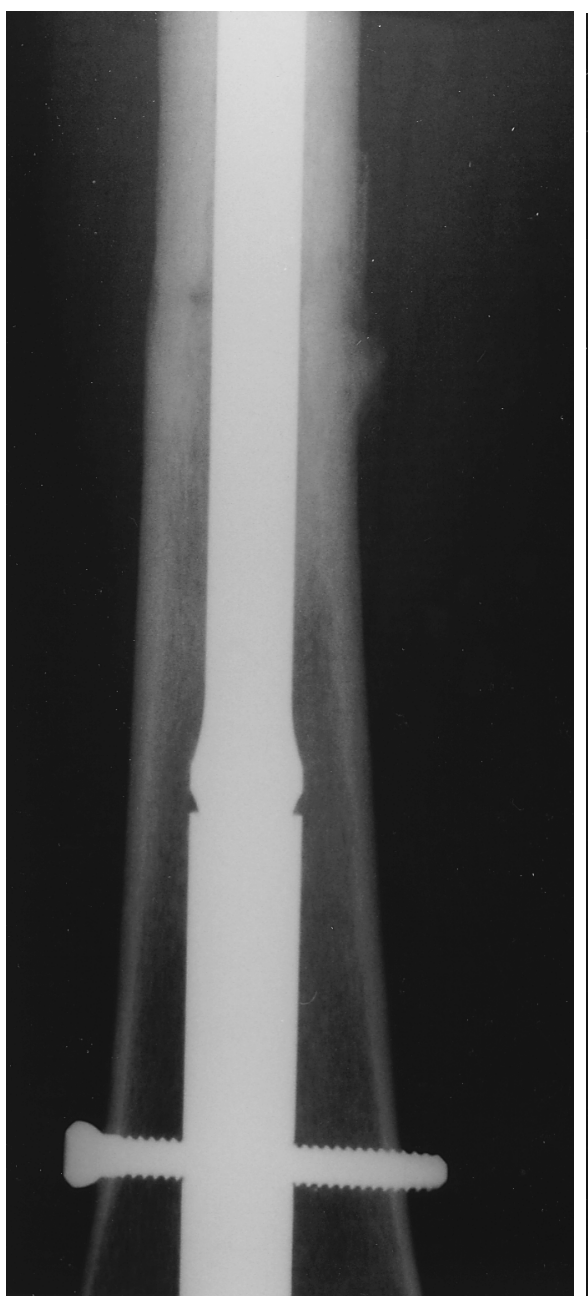

Fig. 4-A

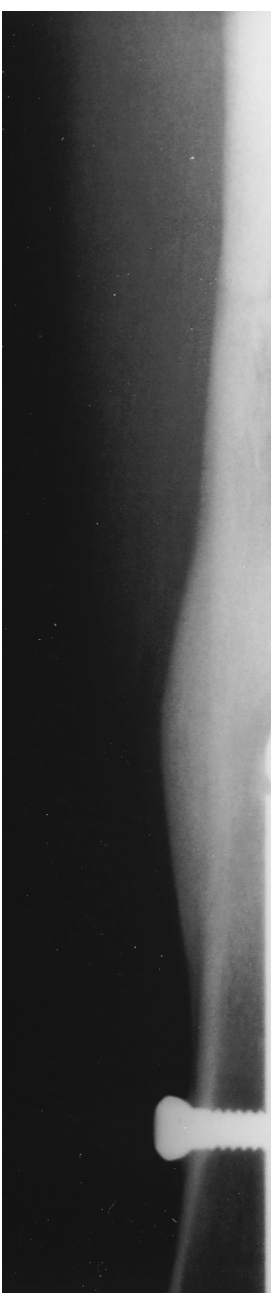

Fig. 4-B

Figs. 4-A and 4-B Case 5. Example of cortical thickening adjacent to the distal, large junction in a nail inserted through a retrograde approach.

Fig. 4-A Radiograph made nine months postoperatively, showing a well-healed fracture and no findings at the junction. Fig. 4-B Radiograph made fifteen months following the index operation, revealing cortical thickening measuring $15 \mathrm{~mm}$. This was preceded by a periosteal reaction.

was $0.03 \mathrm{ng} / \mathrm{mL}$ (parts per billion). Values below the detection limit were assigned a concentration of one-half the detection limit by convention ${ }^{6}$. Serum samples from two of the twentyseven patients had been collected improperly and thus were discarded.

All patients were offered nail removal, and twelve nails were removed. The retrieved nails were analyzed with scanning electron microscopy (model 5900; JEOL, Peabody, Massachusetts) to evaluate the nature of the degradation process at the taper interface.

Peri-implant soft tissues were obtained by curettage of the medullary canal at the level of the modular junctions in eleven patients. The tissues were processed for histologic analysis, and $5-\mu \mathrm{m}$-thick serial sections were cut and stained with hematoxylin and eosin. The sections were examined with light microscopy for cellular infiltrates and the presence of particulate debris. The elemental composition of corrosion products at the modular junctions of the nails and in the histologic sections was determined with electron microprobe analysis (JEOL model JXA 8900) with use of previously described techniques ${ }^{7}$.

A control group of sixteen patients in whom an acute femoral fracture had been treated with a standard one-piece 316L stainless-steel Russell-Taylor intramedullary nail (Smith and Nephew) during the same time-period was also identified. The mean age (twenty-nine years; range, eighteen to fifty-two years) and duration of follow-up (twenty-two months; range, thirteen to forty-two months) of these patients were similar to those of the patients treated with the modular femoral nail. These control patients were selected on the basis of the time of implantation so that the duration of follow-up would be similar to that of the patients with the modular nail. Ten nails had been inserted in an antegrade fashion and six, in a retrograde fashion. Blood samples were obtained for measurement of serum chromium concentration. The pain analog scale and the assessment form were completed. Two of the nails in the control group were removed. Curetted material from the femoral canal was analyzed in the same fashion as it was in the group 
treated with the modular femoral nail. We compared the patients in the control group with the patients treated with the modular nail with regard to serum chromium levels, scores on the pain analog scale, and scores determined from the assessment form and its subscales.

The serum chromium levels in a previously defined ${ }^{6}$ control group of twenty-one patients without a femoral implant were compared with the levels in the patients with the modular femoral nail and those in the control group with the one-piece implant. The group consisted of twelve women and nine men with a mean age of fifty-nine years (range, thirtyfive to seventy-five years).

The patients with the modular femoral nail were divided into two groups on the basis of the severity of the radiographic findings. For this purpose, mild or absent osteolysis was defined as $<2 \mathrm{~mm}$ (twelve patients), and severe osteolysis was defined as $\geq 2 \mathrm{~mm}$ (fifteen patients). Cortical thickening was considered to be mild or absent if it was $<5 \mathrm{~mm}$ (sixteen patients), and it was considered to be severe if it was $\geq 5 \mathrm{~mm}$ (eleven patients). Periosteal reaction was either present (ten patients) or absent (seventeen patients). Eighteen patients with severe osteolysis, cortical thickening, or a periosteal reac-

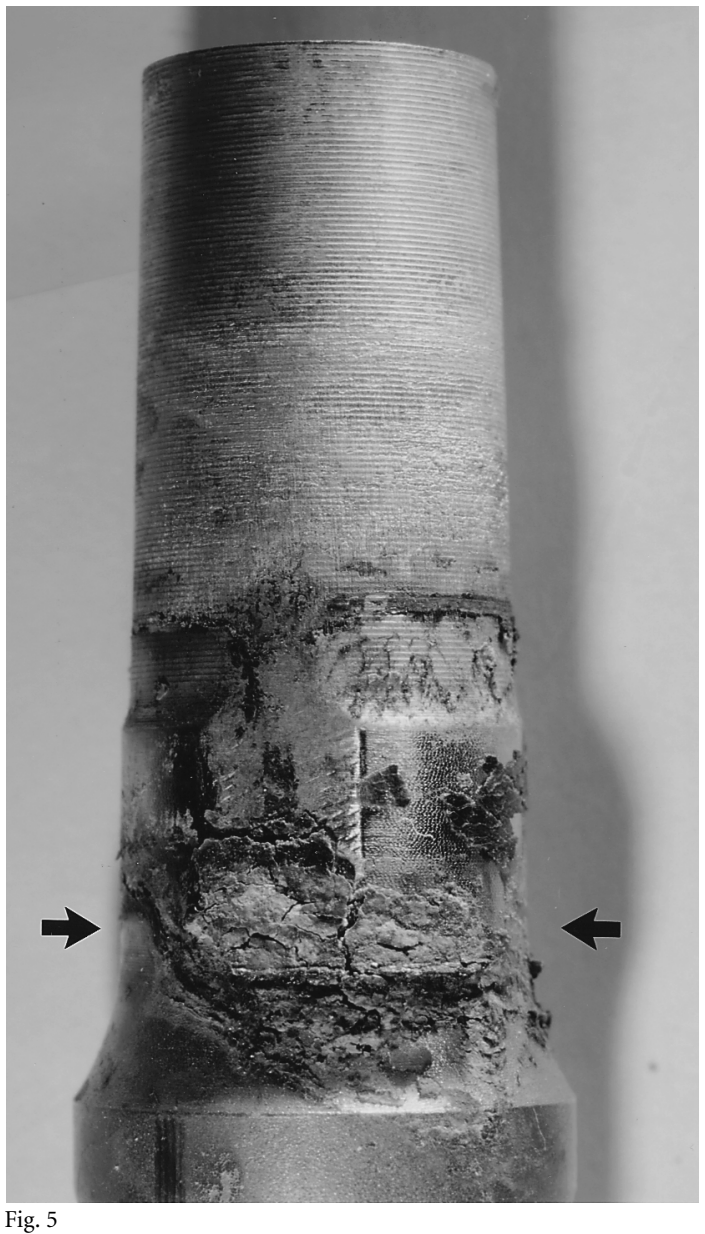

Photograph of a disassembled modular nail, demonstrating adherent corrosion deposits at the opening of the modular junction (original magnification, $\times 5)$. tion at either junction were assigned to group 1 , and nine patients with minimal or no reaction were assigned to group 2. These groups were used to determine if there was an association between the more severe radiographic reactions and the following independent variables: age, gender, fracture type, location of the fracture, location of the modular junction, nail size, method of insertion, tightest isthmal fit of the nail overall and at the large and small modular junctions, proximity of the modular junction to the cortex, dynamic or static locking, and indication for the nailing (an acute fracture or a nonunion). Serum chromium levels and levels of thigh pain were also compared between the two groups.

Group 1 and group 2 were compared with use of the Fisher exact test for the categorical variables (gender, method of insertion, indication for the nailing, type of locking, and type and location of the fracture). A two-sample $t$ test was performed to compare the groups with regard to the age at surgery, and the Wilcoxon two-sample test was carried out to compare the groups with regard to pain, nail size, tightest overall fit, and tightest fit at the large and small modular junctions. The presence of severe findings (osteolysis only, periosteal reaction only, cortical thickening only, or a combination of the three) was compared between the two locations (subtrochanteric and distal) within the same patient with use of the McNemar test. Intergroup comparisons for significant differences in serum chromium levels were performed with use of the Kruskall-Wallis test, followed by a multiple comparison test based on rank sums if the Kruskall-Wallis test indicated a significant difference at the $\mathrm{p}<0.05$ level.

The total scores and the subscale scores on the Musculoskeletal Function Assessment were calculated according to the instructions for administration. This outcomes questionnaire is based on a standardized total score on a scale of 0 to 100 , with 0 indicating no disability and 100 indicating total disability. Intergroup comparisons of the scores were made between the modular nail group and the control group with the one-piece nail with a series of Student $t$ tests with significance set at $\mathrm{p}<0.05$.

\section{Results}

7 wenty-three of the twenty-seven patients (thirteen of the fourteen patients who had had antegrade nailing and ten of the thirteen who had had retrograde nailing) had at least one type of reaction at one or both junctions (Table II). More than one type of reaction was present at the involved junctions in thirteen patients. Osteolysis occurred in nineteen patients and at twenty-three modular junctions (Figs. 3-A and 3-B). The osteolytic lesions were as large as $5 \mathrm{~mm}$, with a mean size of $2.5 \mathrm{~mm}$. The mean time until the osteolysis was first observed was thirteen months (range, three to twenty-nine months). The extent of the osteolysis decreased over time in only three patients. In eight patients, the lesions were either stable (six patients) or they increased (two patients) over time. In another eight patients, the osteolysis was first noticed on the most recent radiographs (at a mean of twenty-two months; range, seventeen to twenty-nine months).

A periosteal reaction occurred in ten patients and at 
thirteen junctions. Seventeen patients (twenty-one junctions) had cortical thickening (Figs. 4-A and 4-B). The mean time until radiographic observation was nine months (range, two to twenty-nine months) for the periosteal reactions and fourteen months (range, five to twenty-seven months) for the cortical thickening. The mean amount of cortical thickening was $6.1 \mathrm{~mm}$ (range, 0 to $15 \mathrm{~mm}$ ). The periosteal reactions preceded cortical thickening in all except two patients. In these two patients, an obvious periosteal reaction that had not been present on radiographs made only several months earlier was seen at the most recent follow-up evaluation, at nineteen and twenty-nine months. The mean amount of cortical thickening following a documented periosteal reaction was $7.2 \mathrm{~mm}$ (range, 0 to $15 \mathrm{~mm}$ ).

All three findings occurred more frequently in the subtrochanteric region than in the distal region, and this difference was significant when only the severe findings were considered $(\mathrm{p}=0.02)$. In the patients who had had antegrade nailing nineteen of the thirty-two reactions involved the large junction, whereas in the patients who had had retrograde nailing nineteen of the twenty-five positive findings occurred at the small junction; both of these junctions were in the subtrochanteric region. With the numbers available, there was no association between the method of insertion and positive radiographic findings. Age, gender, fracture type and location, nail size, tightest overall isthmal fit, tightest fit of the modular junction to the cortex, static or dynamic locking, and whether the procedure was performed for an acute fracture or a nonunion were not found to be significant factors ( $p>0.05)$, with the numbers available, in the development of severe reactions. Four modular femoral nails were dynamically locked (three in a femur with a nonunion). Six of the eight junctions in these nails were associated with a reaction.

The mean serum chromium level (and standard deviation) in samples drawn from twenty-five of the twenty-seven patients with a modular nail was $1.04 \pm 0.57 \mathrm{ng} / \mathrm{mL}$ (range, 0.12 to $3.12 \mathrm{ng} / \mathrm{mL}$ ). This level was significantly higher than the mean level in the control group with the one-piece nail $(0.26 \pm 0.40 \mathrm{ng} / \mathrm{mL}$; range, 0.015 to $1.25 \mathrm{ng} / \mathrm{mL}$ [nondetectable in five patients] $)(\mathrm{p}<0.05)$ and the mean level in the control group without an implant (mean, $0.05 \pm 0.06 \mathrm{ng} /$ $\mathrm{mL}$; range, 0.015 to $0.25 \mathrm{ng} / \mathrm{mL}$ [nondetectable in twelve patients]) $(\mathrm{p}<0.01)$. The mean in group 1 (patients with the modular nail who had more severe radiographic reactions) was $1.27 \pm 0.73 \mathrm{ng} / \mathrm{mL}$, and the mean in group 2 (patients with the modular nail who had mild or no radiographic reactions) was $0.53 \pm 0.41 \mathrm{ng} / \mathrm{mL}$. This difference did not reach significance with the numbers available $(\mathrm{p}<0.90)$. The mean serum chromium level in group 1 was significantly higher than the mean level in the control group with the one-piece implant $(\mathrm{p}<0.01)$ and in the control group with no implant $(\mathrm{p}<0.01)$. Group 2 had a significantly higher mean serum chromium level when compared with the control group with no implant $(\mathrm{p}<0.01)$ but not when compared with the control group with the one-piece nail ( $\mathrm{p}=$ 0.37 ). The difference between the two control groups was not significant $(\mathrm{p}=0.74)$.

The score for thigh pain on the visual analog scale averaged 2.2 (range, 0 to 6 ) in the modular nail group and 0.9 in the control group with the one-piece nail. With the numbers available, this difference was not significant $(p=0.07)$. However, the difference between the patients in group 1 (mean,

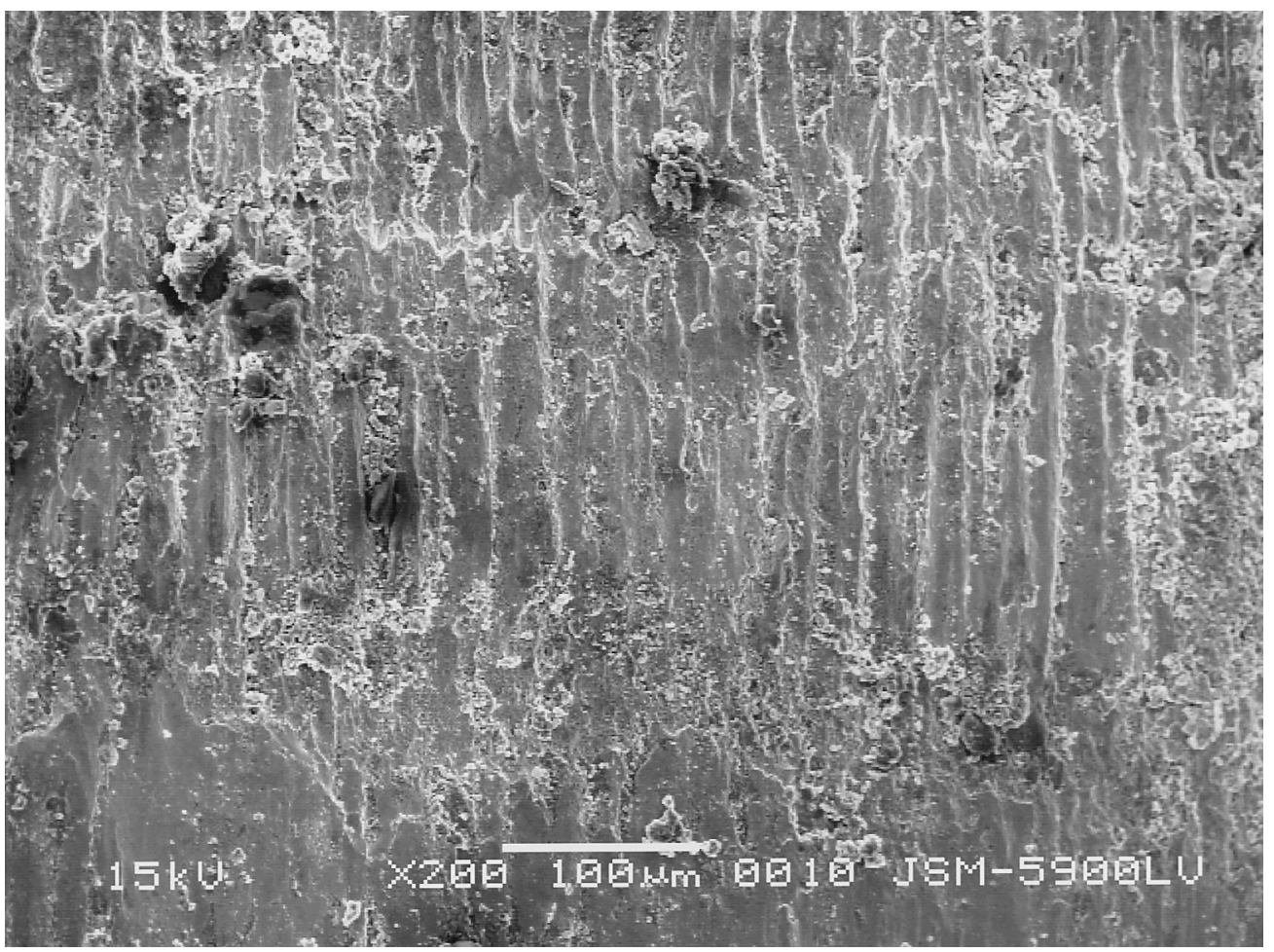

Fig. 6

Scanning electron micrograph of the cone surface, in the taper interface region, of an intramedullary nail. Note the vertical fretting scars and evidence of small corrosion pits (original magnification, $\times 200$ ). 
2.6; range, 0 to 6 ) and the control group was significant ( $\mathrm{p}=$ $0.03)$. There was no significant association between the presence of thigh pain and the serum chromium level $(p=0.45)$. However, six patients with a modular femoral nail had a score for thigh pain of $\geq 5$ on the scale of 0 to 10 . Five of these six patients had osteolysis of $\geq 2 \mathrm{~mm}$, and four had a chromium level of $>0.9 \mathrm{ng} / \mathrm{mL}$.

The mean total score derived from the Musculoskeletal Function Assessment was $22.6 \pm 16.5$ in the modular nail group and $16.5 \pm 14.0$ in the control group with the one-piece nail. These scores and the subscale scores were not found to be significantly different with the numbers available $(\mathrm{p}=0.25)$.

All of the modular junctions of the retrieved nails were grossly intact on manual testing. Corrosion products were visible at the modular junctions of eleven of the twelve modular nails (Fig. 5). Evidence of fretting corrosion was apparent on scanning electron microscopic analysis (Fig. 6). The fretting scars ran parallel with the long axis of the nail and were approximately 100 to $200 \mu \mathrm{m}$ in length.

Backscattered electron micrographs of corrosion debris found in the taper interface, deep inside the crevice, showed that the debris consisted of plates of corrosion product overlaying the alloy. The corrosion plates at this location were found to contain a high quantity of molybdenum relative to the alloy composition. At the opening of the crevice, the corrosion products were different in character and were of two types. The first type was a brownish flaky deposit with a high iron and oxygen content and lesser amounts of chromium. The second was a greenish plate-like deposit rich in chromium, phosphorus, and oxygen.

Tissue specimens curetted from the medullary canal at the levels of the modular junctions consisted of fragments of fibrous membrane and granulation tissue demonstrating chronic inflammation. The cellular infiltrate included abundant, particle-laden macrophages and foci of plasmacytes accompanied by fewer lymphocytes (Fig. 7). There were two types of particles within the macrophages: golden-brown, hemosiderin-like granules, 1 to $3 \mu \mathrm{m}$ in size, and pale-green, plate-like particles, 1 to $5 \mu \mathrm{m}$ in size. Larger particles of the plate-like material, as large as several hundred micrometers, were also present, associated with multinucleated giant cells. Energy-dispersive $\mathrm{x}$-ray analysis indicated that the hemosiderin-like granules were rich in iron and oxygen, with occasional traces of chromium. The greenish, plate-like particles were rich in chromium, phosphorus, and oxygen and had relatively low amounts of iron. Both of these corrosion products in the tissues were similar in composition to the corrosion products isolated from the modular junctions of the rods, surrounding the opening of the crevice. No metal debris was found in the femoral canal membrane of the two patients from whom a one-piece nail had been removed.

\section{Discussion}

Deri-implant osteolysis, which is most commonly associated with total hip replacement, has not, to our knowledge, been previously reported in association with femoral intramedullary nails. We suspect that metal debris from corrosion at the nail junctions was responsible for the local radiographic findings in our patients. The elevated serum chromium levels and the visible corrosion products at the modular junctions and within the tissue adjacent to eleven of the twelve retrieved devices support this assertion. The corrosion that

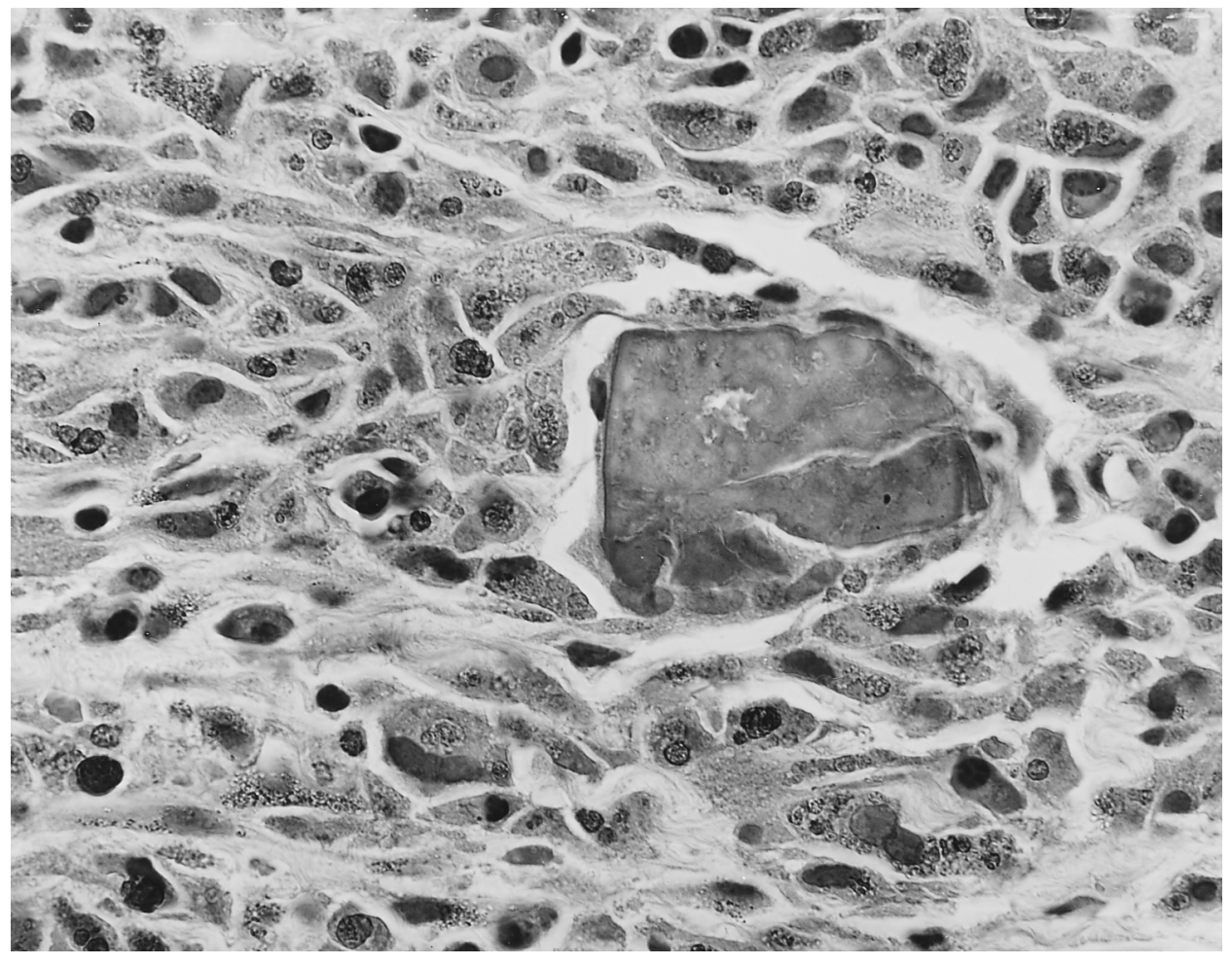

Fig. 7

Case 19. Twelve months following intramedullary nailing of a femoral shaft fracture, granulation tissue surrounding the corroded modular femoral nail demonstrated extensive infiltration of particle-laden macrophages and plasmacytes. Two types of particles were identified: golden-brown, hemosiderin-like granules rich in iron and oxygen with occasional traces of chromium, and pale-green, plate-like particles rich in chromium, phosphorus, and oxygen. A single larger particle of the latter material is seen centrally. Both of these particle types were similar in composition to those demonstrated to be adherent to the surface of the nails, as seen in Figure 5 (hematoxylin and eosin, $\times 750$ ). 
was observed at the taper interfaces of the retrieved nails was similar in character to mechanically assisted crevice corrosion found in total hip femoral stems. Both mechanical fretting and evidence of corrosion were found in our study. The corrosion debris found within the taper, at both deep and superficial locations, appeared to be rich in elements that are less soluble in aqueous electrolyte (for example, molybdenum and chromium) compared with the alloy composition. Again, this finding is similar to that seen at other tapers ${ }^{8}$. Fretting crevice corrosion of the taper interfaces dramatically increases the rate of release of corrosion products as well as the rate of reduction reactions (for example, consumption of oxygen) elsewhere on the surface. The potential of the implant also becomes more negative than its at-rest potential. Each of these factors can affect the performance of the implant. Also, the degradation of the taper interface may mechanically compromise the nail, increasing the risk of device fracture'.

Two types of corrosion products were observed in the peri-implant tissues. These were similar in composition to corrosion deposits at the opening of the taper crevice and similar to particles reported in association with stainless-steel internal fixation devices ${ }^{10,11}$. The first of these products consisted of iron and oxygen-rich particles. While these particles are somewhat similar to hemosiderin deposits, as might be expected at the site of internal fixation of a fracture, the enormous volume of these particles, the time since the fracture fixation, and the fact that traces of chromium were detected in many of these particles strongly suggest that they were a result of stainless-steel corrosion. The second of these products was quite similar in histologic and gross appearance, as well as in elemental composition, to the chromium orthophosphate hydrate-rich corrosion product reported in association with both similar-metal and mixed-metal modular femoral total hip-replacement components ${ }^{7,12-14}$.

There is evidence that these chromium orthophosphate hydrate-rich corrosion products may cause osteolysis not only by increasing polyethylene wear through a three-body mechanism but also through a cellular response from macrophages leading to bone resorption through a separate mechanism $^{12,15,16}$. Metal debris has been shown to stimulate osteoclast production leading to bone resorption ${ }^{17}$. In the published literature on arthroplasty-associated osteolysis, it has not been possible to isolate the effects of particulate articular wear debris, cement debris, and metallic corrosion debris. The modular femoral nail provides a unique system whereby the local tissue effects of metallic corrosion debris alone can be observed. Femoral osteolysis was seen in association with metallic corrosion products in the absence of polyethylene, polymethylmethacrylate, or other debris typically seen with joint arthroplasty reconstructions. This observation provides additional evidence that at least some cases of osteolysis following total hip arthroplasty may be the result of the generation of metallic corrosion products at modular junctions.

While corrosion is unusual with one-piece internal fixation devices ${ }^{13,18}$, a positive association has been demonstrated between the presence of corrosion and tissue reac- tion around multiple-piece stainless-steel implants ${ }^{19-21}$. These findings have been noted at a plate-and-screw interface that has a substantially different geometry than the tight-fitting taper junctions of the modular femoral nail. Also, the junctions between plates and screws are small, extracortical, and away from the bone. Therefore, it is unlikely that the small amount of corrosion products that may develop would stimulate a localized cellular reaction substantial enough to cause a reaction of the same magnitude that we have seen. The device reported on in the present study has two large, intracortical junctions where such fretting and corrosion could have occurred and stimulated the type of cellular response necessary to cause osteolysis.

The corrosion process at modular junctions of femoral total hip components has been hypothesized to occur as a result of a combination of cyclic relative motion, known as fretting, and crevice corrosion, which is the electrochemical process that occurs after the breakdown of the passivating oxide film in an environment with a restricted flow of oxy$\operatorname{gen}^{13,20,22}$. Spindle-shaped cortical thickening and scalloping osteolysis secondary to corrosion and abrasion of cemented titanium femoral stems have recently been reported at postoperative intervals (cortical thickening at twenty-four months and osteolysis at thirty-five months) similar to those in our study $^{16}$. It is likely that a similar mechanism of accelerated corrosion occurred at the taper junctions in the three-piece nails in our patients. The cellular response to the metal debris and corrosion products, in turn, resulted in focal osteolysis. Except for the series of cemented titanium stems discussed above ${ }^{16}$, it appears that the osteolysis observed in association with modular femoral nails occurred earlier than is typically reported for hip arthroplasty. The reasons for this are not clear but could be related to a higher local concentration of corrosion products, the unique chemistry of the corrosion products, the proximity (and intraosseous location) of the modular femoral nail junctions to the cortical surface of the femur (modular head-neck junctions of femoral total hip components are in an extraosseous location), or a combination of these factors.

In every case but two, periosteal reaction preceded cortical thickening. It is likely, therefore, that periosteal reaction and cortical thickening occur along a continuum. The presence of a periosteal reaction was also strongly associated with osteolysis. The periosteal reaction and cortical thickening possibly resulted from increased bone formation through injury and repair following the cellular mediated bone resorption that occurs as a result of the corrosion products.

The association of these radiographic findings with thigh pain is of clinical interest. The patients in group 1, who had the more severe reactions, had a significantly higher score for thigh pain than did our control patients with a one-piece nail $(\mathrm{p}=0.03)$. Thigh pain was the presenting symptom in the first two patients whom we observed to have radiographic reactions. Both had a large area of osteolysis and a periosteal reaction at modular junctions well away from the healed fracture. Also, $20 \%$ of the patients with the modular femoral nail described pain that limited activities of daily living. In 
their series of patients in whom cortical thickening and osteolysis developed following primary implantation of a cemented titanium total hip replacement, Willert et al. described the early onset of dull, constant thigh pain, which was also thought to be associated with similar radiographic findings ${ }^{16}$.

In two of our patients with marked osteolysis and thigh pain (Cases 7 and 19), nail removal resulted in relief of the thigh pain, an observation that further implicates corrosion products as the cause of symptomatic osteolysis.

The time of appearance of these findings has been unpredictable, ranging from two to twenty-nine months after injury. Of the many factors evaluated, only the location of a nail junction in the subtrochanteric region appeared to have an association with the prevalence of severe reactions. The increased stresses in this area may have resulted in more extensive fretting, facilitating the process of mechanically assisted crevice corrosion ${ }^{22}$.

Circulating levels of metals, particularly chromium, have been associated with corrosion at modular junctions in patients with cobalt-chromium-alloy implants ${ }^{6,12}$. In our study, the mean level of serum chromium in the patients with the modular femoral nail was significantly higher than that in our control group with a one-piece stainless-steel intramedullary nail $(\mathrm{p}<0.01)$ and that in our control group without an implant $(\mathrm{p}<0.01)$. While the clinical impact of elevated serum levels of chromium and their association with osteolysis have not been fully defined, serum chromium levels may well serve as a marker for the degree of fretting corrosion at the modular junctions. This has also been suggested to be the case for fretting corrosion at the modular junctions of cobalt-chromiumalloy total hip replacements ${ }^{6,12}$.

In summary, we concluded that a biological reaction to fretting corrosion products around the junctions of this modular femoral nail caused the radiographic findings of osteolysis, periosteal reaction, and cortical thickening; the elevated serum chromium levels; and the thigh pain in our patients. As a result of these findings, we have abandoned the use of this device, and it was withdrawn from the other institutions participating in the clinical evaluation. Surgeons and designers of fracture implants must be certain that the benefits of modularity are not offset by the problems related to load-bearing metal-metal junctions. To realize the benefits of modularity, careful preclinical laboratory evaluation of modular devices with use of relevant in vitro simulations must be performed ${ }^{23}$.

Darron M. Jones, MD

J. Lawrence Marsh, MD

James V. Nepola, MD

Joseph A. Buckwalter, MD

Department of Orthopaedic Surgery, the University of Iowa College of Medicine, Lower Level, JPP, Iowa City, IA 52242. E-mail address for J.L. Marsh: j-marsh@uiowa.edu

Joshua J. Jacobs, MD

Anastasia K. Skipor, MS

Robert M. Urban

Rush Arthritis and Orthopaedics Institute, Rush-Presbyterian-St. Luke's Medical Center, 1653 West Congress Parkway, Chicago, IL 60612. E-mail address for J.J. Jacobs: jacobs@orth4.pro.rpslmc.edu

Jeremy L. Gilbert, $\mathrm{PhD}$

Department of Bioengineering and Neuroscience, Syracuse University, Syracuse, NY 13244

Although none of the authors has received or will receive benefits for personal or professional use from a commercial party related directly or indirectly to the subject of this article, benefits have been or will be received, but are directed solely to a research fund, foundation, educational institution, or other nonprofit organization with which one or more of the authors is associated. Funds were received in total or partial support of the research or clinical study presented in this article. The funding sources were Smith and Nephew Richards, Incorporated; National Institutes of Health/National Institute of Arthritis and Musculoskeletal and Skin Diseases Grant 39310; and Crown Family Chair of Orthopaedic Surgery.

\section{References}

1. Jones DM, Marsh JL. Osteolysis of the femur at the junction of a modular intramedullary nail. A case report. lowa Orthop J. 1998;18:136-41.

2. Gustilo RB, Anderson JT. Prevention of infection in the treatment of one thousand and twenty-five open fractures of long bones: retrospective and prospective analyses. J Bone Joint Surg Am. 1976;58:453-8.

3. Fracture and dislocation compendium. Orthopaedic Trauma Association Committee for Coding and Classification. J Orthop Trauma. 1996;10:Supp 1:v-ix, 1-154.

4. Engelberg R, Martin DP, Agel J, Obremsky W, Coronado G, Swiontkowski MF. Musculoskeletal Function Assessment instrument: criterion and construct validity. J Orthop Res. 1996;14:182-92.

5. Martin DP, Engelberg R, Agel J, Snapp D, Swiontkowski MF. Development of a musculoskeletal extremity health status instrument: the Musculoskeletal Function Assessment instrument. J Orthop Res. 1996;14:173-81.

6. Jacobs JJ, Skipor AK, Patterson LM, Hallab NJ, Paprosky WG, Black J, Galante JO. Metal release in patients who have had a primary total hip arthroplasty. A prospective, controlled, longitudinal study. J Bone Joint Surg Am. 1998;80:1447-58

7. Urban RM, Jacobs JJ, Gilbert JL, Galante JO. Migration of corrosion products from modular hip prostheses. Particle microanalysis and histopathological findings. J Bone Joint Surg Am. 1994;76:1345-59.

8. Gilbert JL, Buckley CA, Jacobs JJ. In vivo corrosion of modular hip prosthesis components in mixed and similar metal combinations. The effect of crevice, stress, motion, and alloy coupling. J Biomed Mater Res. 1993;27:1533-44.

9. Gilbert JL, Buckley CA, Jacobs JJ, Bertin KC, Zernich MR. Intergranular corrosion-fatigue failure of cobalt-alloy femoral stems. A failure analysis of two implants. J Bone Joint Surg Am. 1994;76:110-5.

10. Sevitt S. Bone repair and fracture healing in man. New York: Churchill Livingstone; 1981. Corrosion of implants and tissue metallosis; p 281-95.

11. Winter GD. Tissue reactions to metallic wear and corrosion products in human patients. J Biomed Mater Res. 1974;8:11-26.

12. Jacobs JJ, Urban RM, Gilbert JL, Skipor AK, Black J, Jasty M, Galante JO. Local and distant products from modularity. Clin Orthop. 1995;319:94-105.

13. Jacobs JJ, Gilbert JL, Urban RM. Corrosion of metal orthopaedic implants. J Bone Joint Surg Am. 1998;80:268-82.

14. Urban RM, Jacobs JJ, Gilbert JL, Rice SB, Jasty M, Bragdon CR, Galante Jo. Characterization of solid products of corrosion generated by modularhead femoral stems of different designs and materials. In: Marlowe DE, Parr JE, Mayor MB, editors. Modularity of orthopedic implants. American Society for Testing and Materials Special Technical Publication 1301. West Conshohocken, PA: American Society for Testing and Materials; 1997. p 33-44

15. Lee SH, Brennan FR, Jacobs JJ, Urban RM, Ragasa DR, Glant TT. Human monocyte/macrophage response to cobalt-chromium corrosion products and 
titanium particles in patients with total joint replacements. J Orthop Res. 1997;15:40-9.

16. Willert HG, Broback LG, Buchhorn GH, Jensen PH, Koster G, Lang I, Ochsner P, Schenk R. Crevice corrosion of cemented titanium alloy stems in total hip replacements. Clin Orthop. 1996;333:51-75.

17. Kadoya Y, Revell PA, Kobayashi A, al-Saffar N, Scott G, Freeman MA. Wear particulate species and bone loss in failed total joint arthroplasties. Clin Orthop. 1997;340:118-29.

18. Black J. Does corrosion matter? [editorial]. J Bone Joint Surg Br. 1988;70:517-20.

19. Cook SD, Thomas KA, Harding AF, Collins CL, Haddad RJ Jr, Milicic M, Fischer WL. The in vivo performance of 250 internal fixation devices: a follow-up study. Biomaterials. 1987;8:177-84.
20. French HG, Cook SD, Haddad RJ Jr. Correlation of tissue reaction to corrosion in osteosynthetic devices. J Biomed Mater Res. 1984;18:817-28.

21. Williams DF, Meachim G. A combined metallurgical and histological study of tissue prosthesis interactions in orthopedic patients. J Biomed Mater Res. 1974;8:1-9.

22. Gilbert JL, Jacobs JJ. The mechanical and electrochemical processes associated with taper fretting crevice corrosion: a review. In: Marlowe DE, Parr JE, Mayor MB, editors. Modularity of orthopedic implants. American Society for Testing and Materials Special Technical Publication 1301. West Conshohocken, PA: American Society for Testing and Materials; 1997. p 41-50.

23. Jani SC, Reynolds T, Russell A. An improved method for testing fretting corrosion in modular taper junctions. Trans Orthop Res Soc. 1999;24:526. 